\title{
OSTEOLYTIC BONE SYPHILIS
}

\author{
Henry McGladdery, Singapore \\ From the General Hospital, Singapore
}

Bone lesions typical of syphilis include the periosteal node, diffuse osteoperiostitis, and the gumma. Standard descriptions of these syphilitic lesions emphasize that new bone formation usually exceeds bone destruction; but in the cases here reported osteoporosis was the striking feature, and it was much greater than the new bone formation.

\section{CASE REPORTS}

Case 1. Indian woman, aged 25 years-Four years before admission noticed swelling over the inner end of the right clavicle extending gradually over the shoulder and upper arm, associated with aching pain made worse by movement. She had one living child; there had been two miscarriages, one at two months and one at three months. On examination there was generalised swelling and tenderness over the right upper chest and shoulder. The clavicle was not palpable because of the swelling. Movements of the shoulder were full but painful. The Wassermann and Kahn tests were strongly positive. Radiographs showed gross destruction of the clavicle; faint mottling in the middle third of the bone was all that remained (Fig. 1). Radiographic changes were seen also in the first rib and in the humerus; the anterior half of the first rib was destroyed; in the humerus there was destruction of two-thirds of the thickness of the shaft, and this lesion, by itself, resembled a bone tumour. Treatment-The patient was given three and a half million units of penicillin by three-hourly injections over a period of eight days. The pain was relieved, the shoulder felt stronger, and the patient left hospital. Ten months later there were no symptoms. The Kahn test was then negative and radiographs showed that the lesions had healed (Fig. 2).

Case 2. Malay man, aged 25 years-Complained of pain and swelling of the left shoulder of three months' duration. On examination there was a tender swelling over the inner half of the clavicle. There were two large flat paper-like scars on the forearm, said to be the result of large ulcers which had developed several years earlier and had healed spontaneously. The patient gave no history of chancre or rash. He was familiar with yaws but said that he had never suffered from it. The blood Wassermann and Kahn tests were strongly positive. Radiographs showed erosion of the inner quarter of the left clavicle and destruction of the outer end. Neoarsphenamine was given intravenously and potassium iodide by mouth. The pain was relieved within seven days.

Case 3. Chinese male, aged 21 years-Complained of multiple discharging ulcers over the left shoulder. One year before admission noticed redness and tenderness over the upper part of the left shoulder, and then an ulcer which discharged pus; the ulcer and sinus healed but new openings appeared. As one ulcer healed a new one formed behind it, leaving a thin white scar. On examination the inner ends of both clavicles were enlarged and tender (Fig. 3). There was a flat scar with an ulcerating advancing edge (Fig. 4) and the history, clinical appearances and positive Wassermann and Kahn tests left no doubt that this was a gummatous ulceration of the skin. Radiographs showed areas of bone destruction in both clavicles (Fig. 5). As a therapeutic test the patient was put on potassium iodide, thirty grains thrice daily by mouth, and neoarsphenamine intravenously. Healing of the ulceration began in three weeks and was complete in six weeks, thus confirming that the lesions were probably syphilitic. A course of three and a half million units of penicillin was given. The swelling and tenderness disappeared.

Case 4. Chinese woman, aged 25 years-Two months' history of painful swelling of the left foot, increasing in size. The pain was never severe. On examination there was a red and tender swelling over the medial side of the dorsum of the foot with oedema. Radiographs showed an osteolytic lesion of the first metatarsal with pathological fracture. The blood Kahn test was strongly positive. Plaster was applied from the metatarsal heads to the tibial tuberosity, and anti-syphilitic treatment was instituted. Two months later, radiographs showed union of the fracture and recalcification of the bone. Radiographs four months later showed final consolidation.

Case 5. Chinese man, aged 32 years-Intermittent fever and rigors for three months before admission; six weeks before had fallen while walking and fractured the left tibia and fibula; one week before admission had been trying to walk but again fell and fractured the right femur. On examination he looked ill and emaciated. There was gummatous ulceration of the oral pharynx and soft palate with old scars and adhesion of the left half of the soft palate to the posterior pharyngeal wall. The penis showed scarring of the glans 


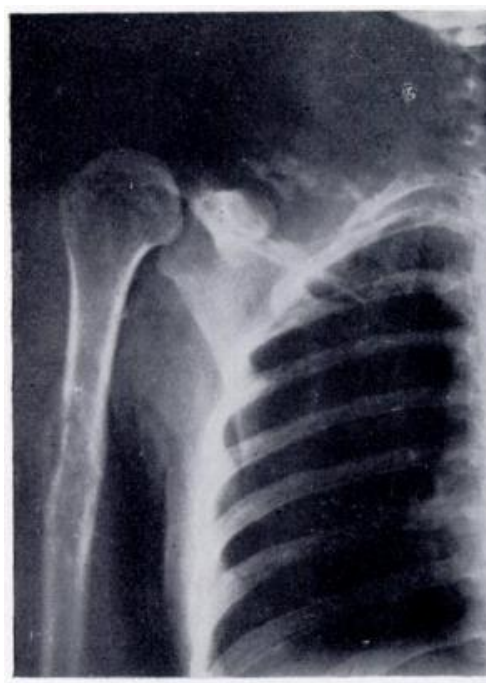

FIG. 1

Case 1. Syphilitic lesions of the right clavicle, first rib and humerus. The clavicle is represented only by faint mottling in the middle third

(Fig. 1). Ten months later, after treatment, the lesions are healed (Fig. 2).

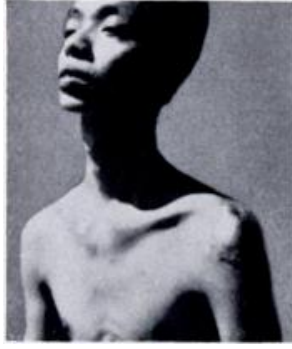

FIG. 3

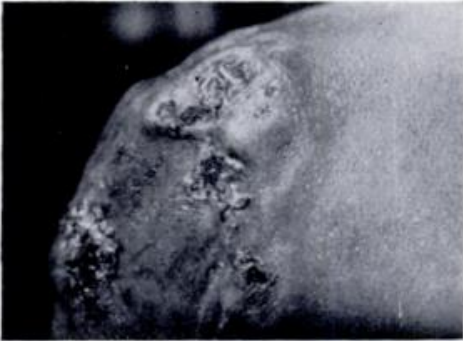

FIG. 4

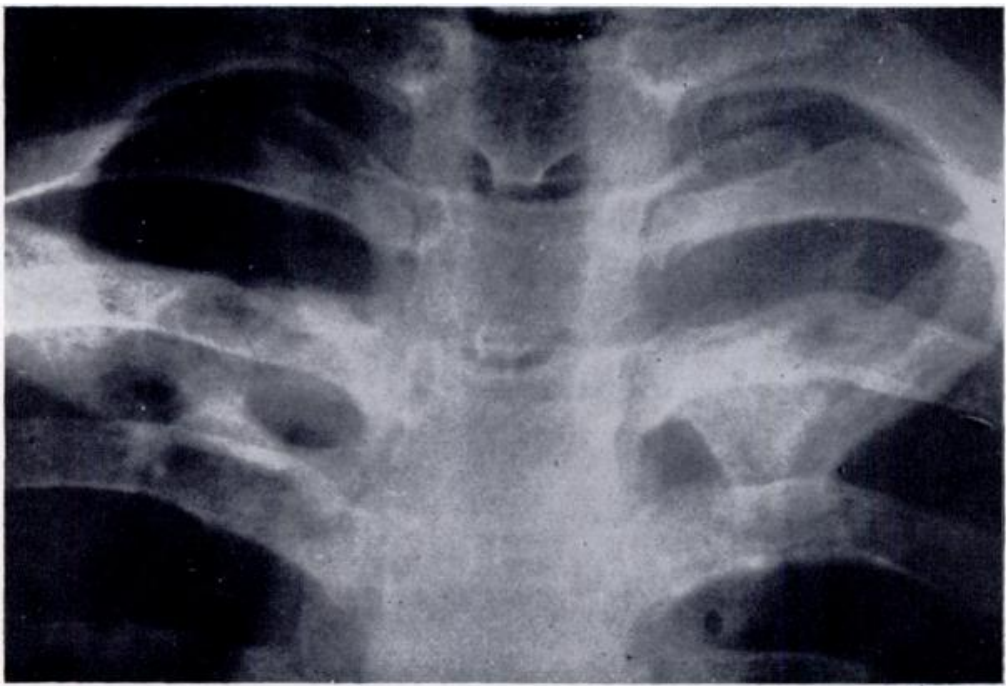

FIG. 5

Case 3. (Figs. 3, 4 and 5.) Syphilitic lesions of the inner ends of both clavicles with gummatous ulceration of the skin over the back of the left shoulder. 
and constriction of the external meatus. In the lower limbs there was extreme wasting of all the muscles. The upper end of the left tibia was irregularly enlarged, hard and tender. There was an obvious fracture of the mid-shaft of the right femur. Radiographic examination-There were fractures of the tibia and fibula in their upper thirds; the whole tibia was diseased, the shaft being speckled with small clear lesions. There was an area of cortical erosion just above the fracture and several areas of demarcated bone destruction in the shaft. Periosteal thickening extended throughout the length of the bone. At the fracture-site there was sclerosis and callus formation and both bones showed evidence of union. In the femur there was a fracture of the middle third through the site of an extensive osteolytic lesion (Fig. 6).

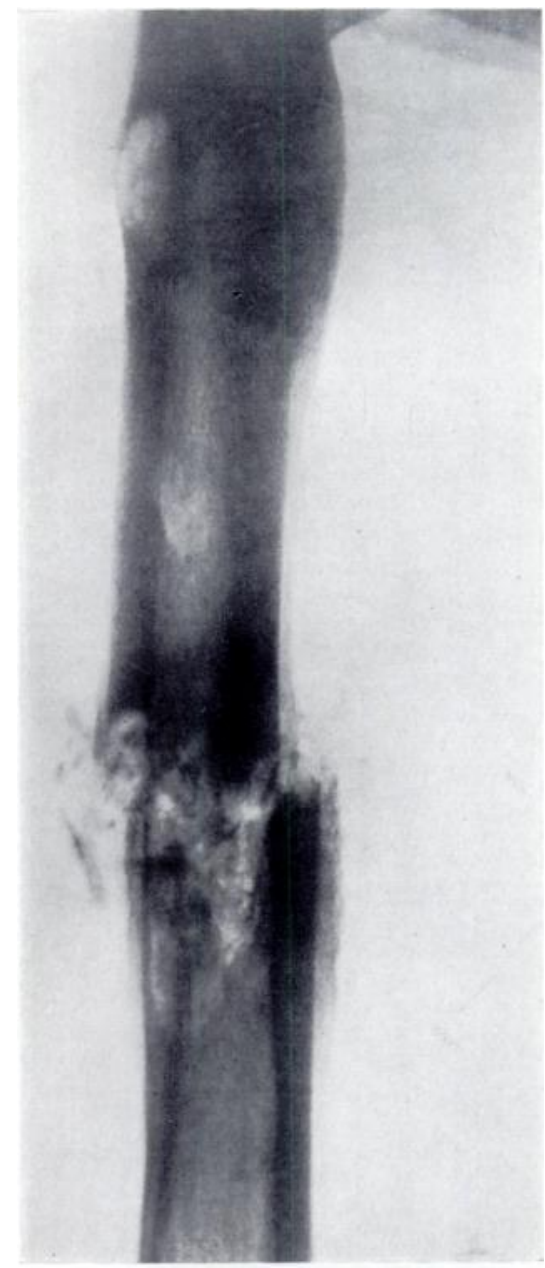

FIG. 6

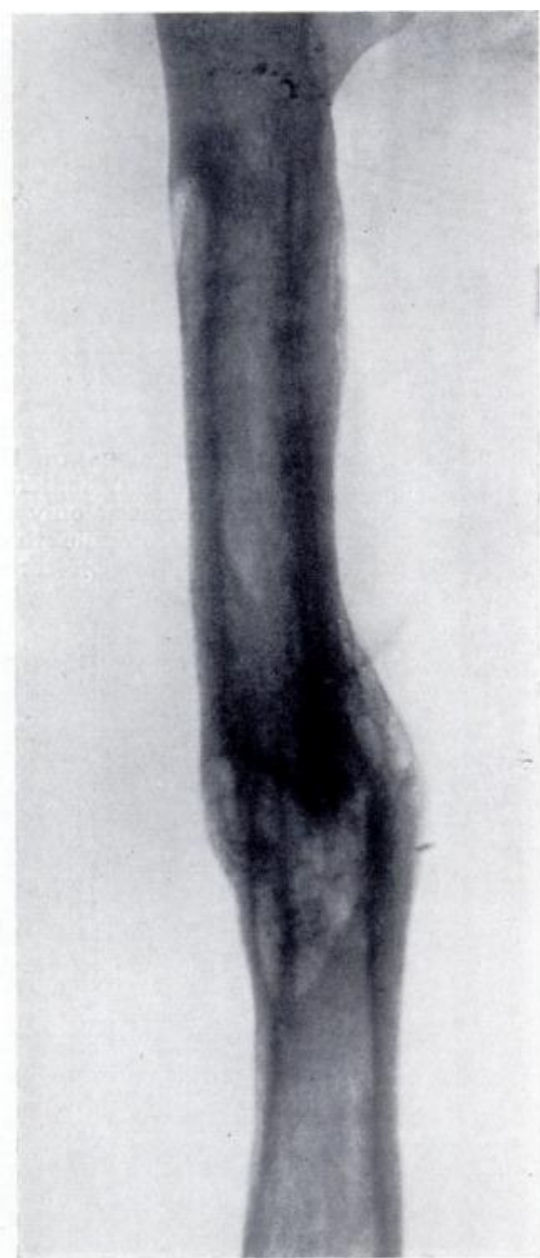

FIG. 7

Case 5. Pathological fracture of the femur through the site of an osteolytic siphilitic lesion; there is also a periosteal node below the lesser trochanter and other areas of bone destruction in the shaft (Fig. 6). After immobilisation in a Thomas's splint with anti-syphilitic treatment the bone lesions healed and the fracture united (Fig. 7).

There were areas of bone destruction in the shaft of the bone above the fracture with a typical periosteal node just below the lesser trochanter. The blood Kahn test was strongly positive. The limb was immobilised in a Thomas' splint and anti-syphilitic treatment was given. The general condition of the patient improved rapidly and the fracture united within four months (Fig. 7).

\section{DISCUSSION}

Standard text-books of pathology do not describe destructive șphilitic lesions in long bones and few radiologists recognise them. In the young adult with acquired syphilis the osseous involvement is usually atrophic, whereas in older patients who have had the disease 
for many years the manifestation is nearly always hypertrophic (Stewart 1938). All the patients in this paper were young, their ages ranging from twenty to thirty years, and the lesions could be described as atrophic. Such atrophic syphilitic lesions of bone may cause pathological fracture as in Case 5 of this series and in the case reported by Jackson Burrows (1937).

Osteolytic syphilis of the tibia was described by Ungerman, Vicary and Eldridge (1938); radiographs showed multiple destructive lesions in the cortex of the tibia without periosteal or medullary changes, and the lesions disappeared after anti-syphilitic treatment. The present writer has seen a similar case with destructive syphilitic lesions of the tibia, talus and calcaneus, closely resembling secondary carcinomatous deposits. The diagnosis may be difficult when the lesions are osteolytic. The radiographic appearances in the humerus in Case 1 of this series resembled those of a bone sarcoma; and the localised punched-out lesions may be confused with the secondary deposits of malignant disease. Biopsy is often inconclusive and blood tests are not always positive, so that amputation may even be considered in the mistaken belief that the lesion is a bone tumour. In doubtful cases it is wise to use penicillin as a therapeutic test. If the lesion is syphilitic, symptomatic relief will occur within seven to fourteen days.

I thank Dr Vickers, Director of Medical Services, Singapore, for his permission to publish this article.

\section{REFERENCES}

Burrows, H. J. (1937): Pathological Fracture of Humerus complicating Late Secondary Syphilis. British Journal of Surgery, 24, 452.

STEWART, D. M. (1938): Roentgenological Manifestations in Bone Syphilis. American Journal of Roentgenology, 40, 215.

Uxgerman, A. H., Vicary, W. H., Eldridge, W. H. (1938) : Luetic Osteitis simulating Malignant Disease. American Journal of Roentgenology, 40, 225. 